\title{
Disturbing effect of lockdown for COVID-19 on the incidence of infective endocarditis: a word of caution
}

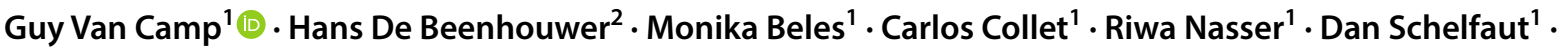 \\ Martin Penicka ${ }^{1}$
}

Received: 13 May 2020 / Accepted: 3 June 2020 / Published online: 10 June 2020

(c) Springer-Verlag GmbH Germany, part of Springer Nature 2020

Sirs:

At the end of December 2019, Chinese authorities reported several cases of acute respiratory syndrome in Wuhan City [1-3]. Also possible impact of underlying cardiovascular diseases on the prognosis of the respiratory syndrome were published [4, 5]. Since then, the COVID-19 virus spread all over the world and the Belgian government decided, as in other countries, to install a lockdown making only essential displacements possible and limiting consultations and interventions to only urgent and necessary indications, thereby limiting considerably the access to usual non-COVID 19 medical care. In summary, authorities gave one major advice: stay at home! At the same time, the major flu-like symptoms of COVID-19 pulmonary infections were widely communicated: fever, dyspnea and cough.

Infective endocarditis (IE) is a serious disease with a high mortality and morbidity and its incidence and severity remain unchanged despite diagnostic and therapeutic improvements [6]. Early diagnosis and treatment is key to avoid major complications as embolic events and local extension of the infection with destruction of the valvular and peri-valvular tissue. Being alert to symptoms and signs of patients susceptible for IE is a prerequisite for this early strategy. However, the main symptoms of fever, dyspnea and cough in a critical patient can be easily mistaken for COVID-19 symptoms although they are also eye-openers in the diagnosis of IE.

Since the initial publications on COVID-19 and the actual worldwide spread of the disease, more than 3.3 million

Guy Van Camp

guy.van.camp@olvz-aalst.be

1 Heart Center OLV Aalst, Moorselbaan 164, 9300 Aalst, Belgium

2 Department of Microbiology, OLV Aalst, Moorselbaan 164, 9300 Aalst, Belgium coronavirus cases and 237,000 deaths are reported. These numbers create inherent fear in the population and together with the terrible images on television of overcrowded hospitals and governments advising staying home, it leads to aversion for hospitalization. This has been reflected in the number of patients presenting with acute myocardial infarction. Several countries have reported a reduction in approximately $40 \%$ in the number of myocardial infarction cases during the COVID-19 lockdown [7, 8].

Taking into consideration these aspects of COVID-19 and IE, and being afraid of a collateral damage of lockdown isolation on the detection of IE, we evaluated in our hospital the incidence of IE during the lockdown period and compared this with the period before, using our in hospital registry of IE.

Patient population: Single center prospective registry of IE. The registry on IE started in 2015. From that moment the care for our endocarditis patients remained uniform including the liberal use of multimodality imaging when necessary [transthoracic (TTE), transoesophageal echocardiography (TOE), magnetic resonance imaging and Positron emission tomography/computer tomography].

The study protocol was performed in accordance with the Ethics Committees of our institution. The need for consent to participate in this research study was waived in view of its observational and anonymous nature.

The lockdown period in Belgium for the COVID-19 pandemic started on March 13th 2020. We compared the weekly incidence of definite endocarditis, using the ESC recommendations (Modified Duke Criteria), between the period before and after the lockdown [9]. The pre-lockdown and post-lockdown period included the time period from July 08th 2017 until March 13th (140 weeks) and from March 14th until May 1st 2020 (7 weeks).

Our hospital installed from almost the beginning of the pandemic a strategy of broad Swap PCR testing to all hospitalized patients. Patients with COVID-positive testing 
are admitted to a special unit. Those who necessitate intensive care are admitted in a specially dedicated COVID intensive care unit. Waiting for the results of the Swap PCR, patients are admitted to a specially dedicated preCOVID unit if they have symptoms of acute respiratory disease (fever, cough, dyspnea). If not they are admitted to the appropriate department, this in a single room with droplet infection protection measurements, until the result of the swap is known.

Strategy for TTE and TEE's has changed drastically in our hospital in the Corona pandemic, this in accordance with the recent paper of EACVI [10]. Indications are stricter to "only if really necessary and urgent—cannot be postponed". This is even stricter for TOE since the risk inherent to this procedure. Protection materials are available in the hospital and used in accordance with EACVI recommendations.

The mean number of admissions for definite IE per week before and after the lockdown period were compared using student's $t$ test. The Levene's test for equality was used to assess the presence of equal variance and results are presented accordingly. Generalized linear model corrected for time as a continuous variable was used to estimate the change in the weekly incidence of IE in the period before and after the COVID lockdown. For visual representation (Fig. 1), the number of pre-lockdown period definite IE cases were clustered in periods of 7 weeks and compared with the 7 weeks of lockdown. A second figure (Fig. 2) illustrates the weekly numbers of the latest 21 weeks.
Until April 30th, 11,654 Swap PCR tests were performed in our hospital. From these 11,654, $1784(15,3 \%)$ were positive for COVID-19.

In the 140 weeks preceding the lockdown, 90 patients with IE were admitted in the hospital $(0.64 \pm 0.75 /$ week $)$. Most important patient characteristics are represented in Table 1. During the 7 weeks of lockdown we diagnosed one patient over a period of 7 weeks $(0.14 \pm 0.38$ /week $)$ $(p=0.012)$. Our preliminary analysis during the phase of the COVID pandemic shows an estimated 55\% (95\% CI 6-115) reduction in IE cases.

In Fig. 1 the number of definite IE are represented in clusters of 7 weeks. Figure 2 is a focused image on the latest 21 weeks. Before lockdown, no time period had only one case of IE. Four had 2 cases. Except for hazard, this could also be explained for two of them by the coincidence with holiday - periods, which could be a trigger to postpone hospitalization. The Figures illustrate well the more dramatic decline of the numbers of IE to only one case exactly coinciding with the lockdown period.

During the period before the lockdown, the number of TTE's and TOE's varied from 2819 to 3879 and 215-327 respectively. During the lockdown these numbers decreased to 1071 and 45 respectively. The number of TTE's and TOE's performed during each cluster of 7 weeks is represented in Fig. 1.

One patient diagnosed during the lockdown presented with a late diagnosis. This 51-year-old man had a known

\section{OLV Hospital :Number of admitted IE patients, with the total numbers of performed TTE/TOE $8 / 7 / 2017-1 / 5 / 2020$}

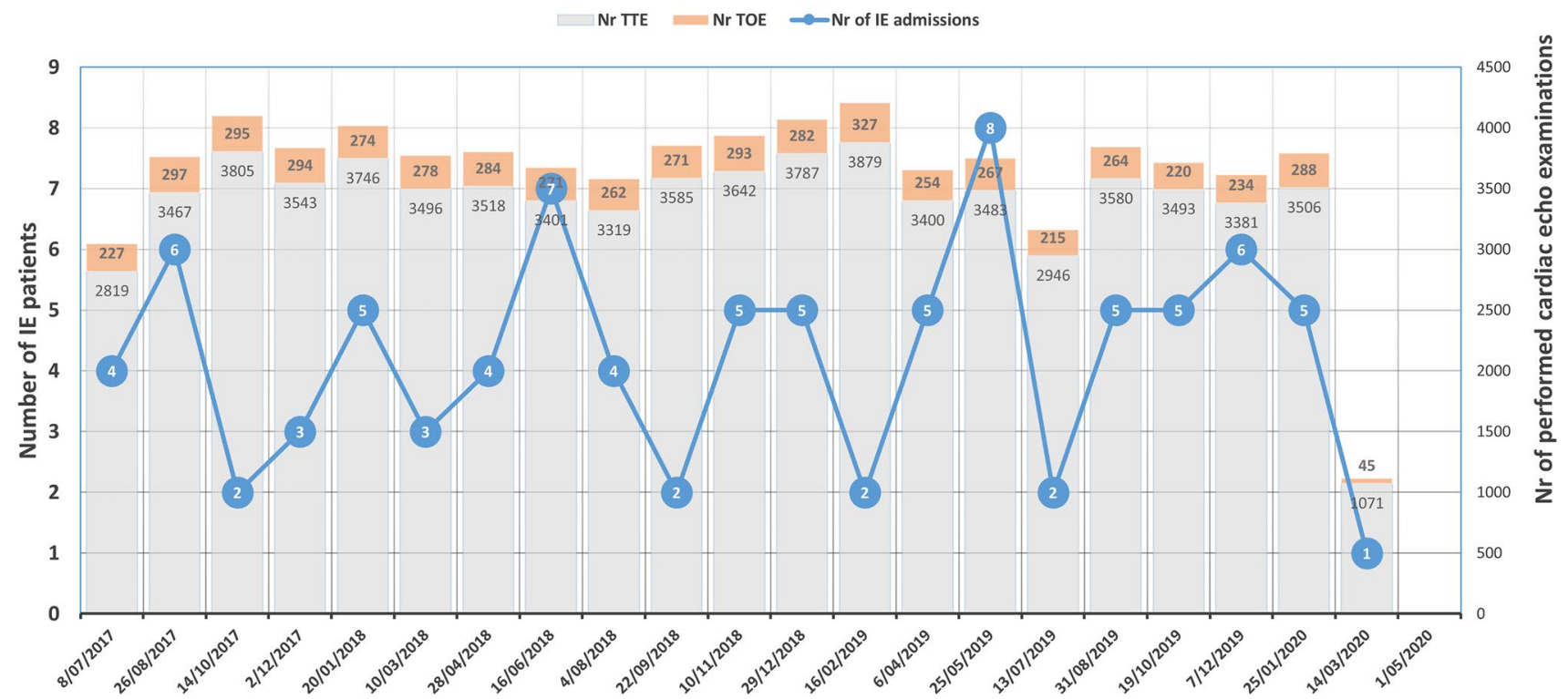

Fig. 1 Graphical representation of the number of patients with definite IE represented by clusters of 7 weeks period. Last cluster of 7 weeks represents the lockdown period in Belgium. Superimposed are the numbers of TTE's and TOE's for each time cluster 


\section{OLV Hospital :Weekly numbers of admitted patients with endocarditis $30 / 11 / 2019-1 / 5 / 2020$ (last 21 weeks of the observation period)}

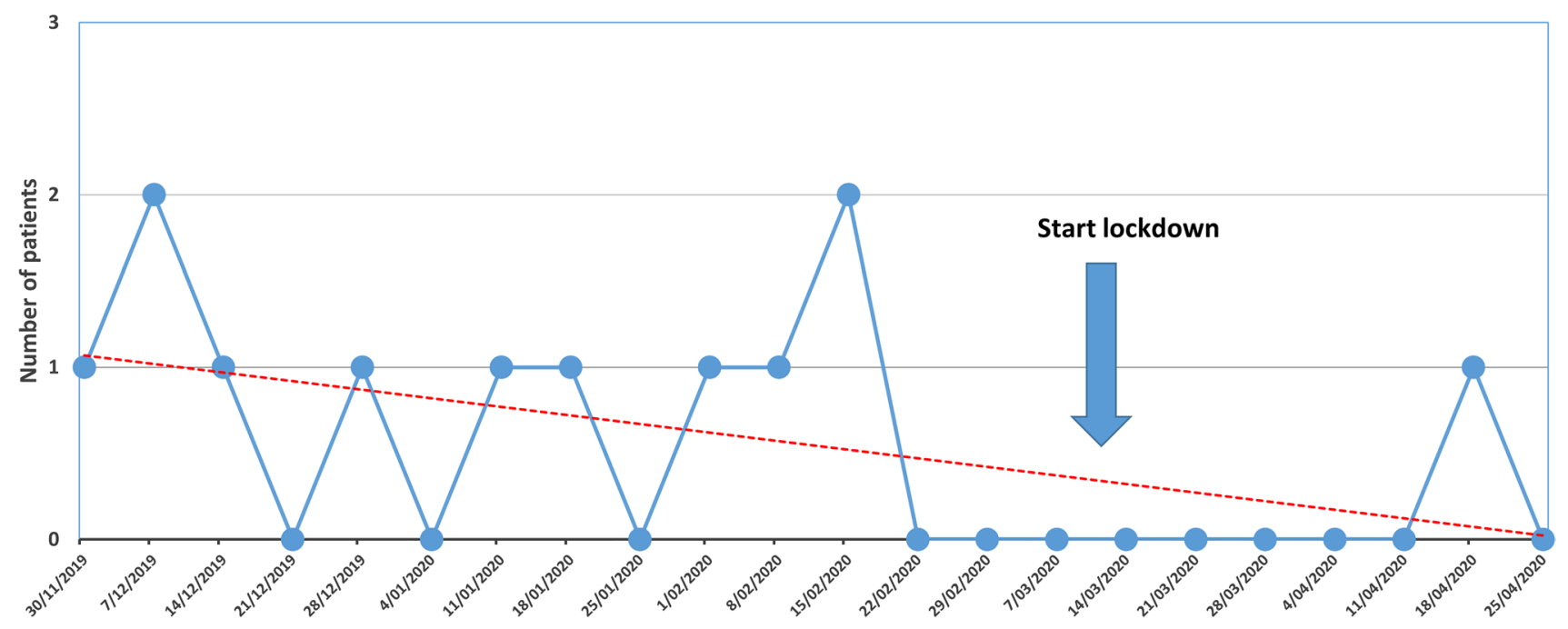

Fig. 2 Focus on the latest 21 weeks. Weekly numbers are shown

Table 1 Patient characteristics

\begin{tabular}{ll}
\hline Patient characteristics & $N=89$ \\
\hline Age (years) & $70.3( \pm 13.9)$ \\
Male & $72 \%$ \\
Prosthetic valve (incl. valvuloplasty) & $48 \%$ \\
ICD/CRT/PM device & $34 \%$ \\
IE type & \\
Native valve & $48 \%$ \\
Prosthetic valve & $44 \%$ \\
Device (ICD/CRT/PM) related & $27 \%$ \\
Microbiologic etiology & \\
Staph. aureus MS & $18 \%$ \\
Staph. aureus MR & $1 \%$ \\
Staph. coagulase negative & $5 \%$ \\
Streptoc. viridans & $10 \%$ \\
Streptoc. bovis & $5 \%$ \\
Pneumococcus & $5 \%$ \\
Enterococcus & $18 \%$ \\
\hline
\end{tabular}

cardiac murmur. He was first referred by his general practitioner to a COVID triage centre with fever and flu-like symptoms. He was sent home in quarantine. After a few days he presented to the emergency department of the hospital with the same complaints and received the same message. Since he developed "blurry" vision he was again referred by his general practitioner for a second time to the emergency department were the diagnosis was made of heart failure, cardiac murmur and a negative $\mathrm{CT}$ of the lungs for COVID-19, and an ischaemic zone was detected in the right cerebellum explaining the vision problems. Haemocultures were positive for Streptococcus parasanguinis and diagnosis was made of an extensive endocarditis of the mitral valve with vegetations on both anterior and posterior leaflet, with pseudo-aneurysm on the anterior leaflet, and massive mitral regurgitation on TTE and TOE. Naso-pharyngeal swab excluded again COVID-19 infection. Urgent surgery, confirming the diagnosis of IE of the mitral valve, was performed after coronary angiography demonstrating a significant lesion on the right coronary artery.

In this manuscript we expose the possible harmful effect of lockdown measures imposed by the government on the detection rate of IE. Although numbers are small, inherent to a mono-centre evaluation of a rather rare disease, the results merit a word of caution towards the medical society and the policy makers. This underdiagnosis of IE might be due to a change in patient behavior, incorrect triage and underuse of the normal diagnostic tools.

Until April 30th, 50,498 positive cases with COVID infection were reported in Belgium. Given the population of Belgium this is a relative big number. This can probably be explained by a return of infected Belgian skiers in the beginning of the COVID pandemic and by the broad testing. This also underlines the appropriateness of the lockdown in Belgium.

IE remains a deadly disease and early diagnosis is the corner stone for early treatment and avoiding unnecessary complications $[6,9]$. The first step for early diagnosis is recognizing the predisposing characteristics of the disease and the symptoms and signs linked to IE. Since fever, shortness of breath and cough are the major symptoms and signs 
of a COVID infection, but also of IE, the risk exists that the COVID pandemic dominates the medical attention towards a "war medicine" approach were these signs leads to quarantine measures and screening pathways by swap-PCR and CT lungs, ignoring possible differential diagnosis.

Even if the physician would stay alert, the first step in a possible diagnosis is a fast presentation at the general practitioner and subsequently at the emergency department. In a period were quarantine is obligatory when signs of fever, cough or dyspnea are present, later presentation of possible IE patients is expected certainly when fear for hospitalisation is collectively present in the population during COVID19 pandemic.

Diagnosis of IE also necessitates a good physical examination followed by the necessary blood cultures and multimodality imaging. Also, these diagnostic tools are often missing in the COVID period. Physical contact necessary for a thorough heart auscultation is often avoided in the emergency department in suspicion of COVID infection. Although swap-PCR and CT lungs are liberally used, haemocultures are not always performed in the patient with fever if the clinical symptoms are not too severe and fever with respiratory complaints are dominating the clinical picture suggesting pulmonary infection eventually by COVID- 19 . The cornerstone for the diagnosis of IE besides haemocultures is and remains echocardiography. In a recent recommendation paper of EACVI, clear restrictions towards the use of TTE and certainly TOE in this COVID-19 period are proposed with obvious protection rules that need to be applied in case TTE and even more TOE is performed [10]. Importantly the paper also gives a clear recommendation for IE patients. The paper correctly alerts physicians that the frequency of IE can rise in this COVID-19 period related to less visits to the dentists and that patients with a suspicion of IE should continue to have a high priority for echocardiography [10]. It is clear however, that the diagnostic barrier imposed by the COVID 19 pandemic, implicates a potential harm for timely diagnosis and treatment in patients with IE.

This report suggests a possible underdiagnosis of definite IE linked to lockdown measures imposed by the authorities in this COVID-19 pandemic. Even if numbers are small implicating a negative impact on strong statistical evidence, the paper gives enough elements to consider this problem and to increase our alertness for timely diagnosis and treatment of this deadly disease even in a COVID-19 pandemic.

\section{Compliance with ethical standards}

Conflict of interest No conflict of interest for all authors.

Ethical standards The study protocol was performed in accordance with the Ethics Committees (EC OLV Aalst 2020/059) of our institution. The need for consent to participate in this research study was waived in view of its observational and anonymous nature.

\section{References}

1. Bogoch A, Watts A, Thomas-Bachli C, Huber MUG, Kraemer KK (2020) Pneumonia of unknown etiology in Wuhan, China: potential for international spread via commercial air travel. J Travel Med. https://doi.org/10.1093/jtm/taaa008

2. Lu H, Stratton CW, Tang YW (2020) Outbreak of pneumonia of unknown etiology in Wuhan China: the mystery and the miracle. J Med Virol 92(4):401-402

3. Zhao S, Lin Q, Ran J, Musa SS, Yang G, Wang W et al (2020) Preliminary estimation of the basic reproduction number of novel coronavirus (2019-nCoV) in China, from 2019 to 2020: a datadriven analysis in the early phase of the outbreak. Int J Infect Dis 92:214-217

4. Li G, Li H, Lu J (2020) No adequate evidence indicating hypertension as an independent risk factor for COVID-19 severity. Clin Res Cardiol. https://doi.org/10.1007/s00392-020-01653-6 [Epub ahead of print]

5. Li B, Yang J, Zhao F, Zhi L, Wang X, Liu L, Bi Z, Zhao Y (2020) Prevalence and impact of cardiovascular metabolic diseases on COVID-19 in China. Clin Res Cardiol 109(5):531-538. https:// doi.org/10.1007/s00392-020-01626-9

6. Habib G, Erba PA, Iung B, Donal E, Cosyns B, Larochel C et al (2019) On behalf of the EURO-ENDO Investigators. Clinical presentation, aetiology and outcome of infective endocarditis. Results of the ESC-EORP EURO-ENDO (European infective endocarditis) registry: a prospective cohort study. Eur Heart J 40:3222-3233

7. Rodríguez-Leor O, Cid-Álvarez B, Ojeda S, Martín-Moreiras J, Rumoroso JR, López-Palop R et al (2020) Impacto de la pandemia de COVID-19 sobre la actividad asistencial en cardiología ntervencionista en España. REC Interv Cardiol 2:82-89

8. Garcia S, Albaghdadi MS, Meraj PM, Schmidt C, Garberich R, Jaffer FA, Henry TD (2020) Reduction in ST-segment elevation cardiac catheterization laboratory activations in the United States during COVID-19 pandemic. J Am Coll Cardiol. https:// doi.org/10.1016/j.jacc.2020.04.011

9. Habib G, Lancellotti P, Antunes MJ, Bongiorni MG, Casalta JP, Del Zotti F, Dulgheru R et al (2015) 2015 ESC Guidelines for the management of infective endocarditis. Eur Heart J 36:3075-3123

10. Skulstad H, Cosyns B, Popescu BA, Galderisi M, Di Salvo G, Donal E et al (2020) COVID-19 pandemic and cardiac imaging: EACVI recommendations on precautions, indications, prioritization, and protection for patients and healthcare personnel. Eur Heart J Cardiovasc Imaging 21:592-598

Funding No funding for this study. 